\title{
Comparison of 2 types of ultrasound guided nerve blocks in patients undergoing breast cancer surgery under opioid free anaesthesia.
}

A Randomised Controlled trial.

Swagata Tripathy, P B Rao, A Panda, Kar M, Mishra T, Nayak S. All India Institute of Medical Sciences, Bhubaneswar, India

\section{INTRODUCTION}

Modified Radical Mastectomy with axillary dissection performed under truncal block without opiods has decreased analgesic requirement, pain scores and PONV compared to opioid based general anesthesia.[1] Recent studies have claimed ultrasound guided PECS block to be better than single level Paravertebral block for this surgery.

\section{OBJECTIVES}

We wished to compare PECS vs single level Paravertebral Blocks (PVB) in an opioid free, nerve block based anesthesia. Outcomes of interest were post operative analgesic requirement, duration of analgesia, PONV, satisfaction of patient and surgeons.

\section{METHODS}

This is a double blind randomized control trial registered in trials registry CTRI/2017/02/007897. Recruiting from October 2016 to June 2017. 58 adult ASA I-III patients posted for MRM-AD, after randomization and allocation concealment with computer generated random numbers obtained over phone, were induced with propofol and maintained on spontaneous ventilation with isoflurane $(0.8-1.0$ MAC) through i-gel. Ultrasound guided PECS or paravertebral blocks $(0.1 \%$ lignocaine $+0.25 \%$ bupivzcaine $+1 \mathrm{mcg} / \mathrm{kg}$ dexmedetomidine, $30 \mathrm{ml}$ ) was administered as per predetermined method. Lack of consent, coagulopathy and allergy to local anesthetics or chronic opioid use were criteria for exclusion. Intraoperative events, post operative pain scores, non opioid analgesic requirement over 24 hours, PONV and satisfaction of surgeon and patient were measured. SPSS 21 was used for analysis. Normality of data was checked by Kolmogorov-Smirnov test and expressed as Mean ( Standard Deviation ) or Median ( Inter quartile Range). Continuous variables were compared by using Student's unpaired t test, and categoric variables by $\chi 2$ test if variables were normally distributedelse, the alternate tests for non-parametric variables were used. The pain scores were considered continuous variable. Confidence intervals were calculated for statistically significant differences

\section{RESULTS}

Between the two groups, there was no difference in demographics, ASA status, location and volume of breast tumour excised or the duration of surgery. There was no difference between the two groups in terms of intra and post operative parameters or block complications. The median VAS scores for pain at rest or during shoulder abduction was similar in both the groups. The time from block to incision was significantly more in the PV group $(p=0.01)$. Satisfaction of surgeons was better in the PECs group.

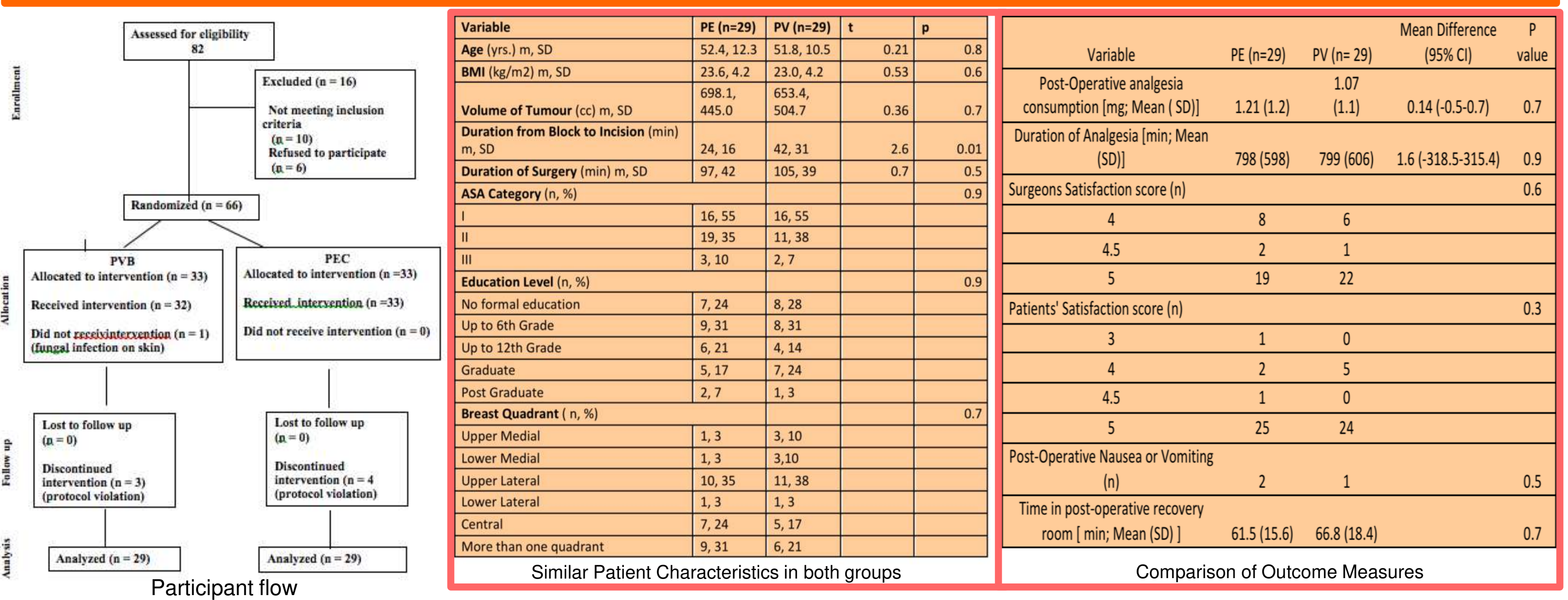

\section{HIGHLIGHTS}

Both the truncal blocks result in adequate prolonged analgesia in the absence of opiate analgesics. (similar to our previous findings [1] and Bashandy et al [2] and unlike Kulhari et al. )

* PECs group demonstrated significantly lesser block-to-incision time .

* Incidence of block related complications was negligible in both groups.

* Avoidance of opioids decreased PONV and post anesthesia recovery times in both groups. No patient required rescue opioids intraoperatively.

* Routine avoidance of opioids with any of these truncal blocks is possible; more evidence is needed for wider acceptance.

1. Tripathy $S$ et al. Opioid-free anesthesia for breast cancer surgery: An observational study. JOACP. 2018.

2. Bashandy GM, Abbas DN. Pectoral nerves I and II blocks in multimodal analgesia for breast cancer surgery.l. Reg Anesth Pain Med. 2015

3. Kulhari S et al. Pectoral nerve block versus thoracic paravertebral block for postoperative analgesia after radical mastectomy. Br J Anaesth. 2016 